\title{
Correlation between homeodomain-interacting protein kinase 2 and apoptosis in cervical cancer
}

\author{
YU CHENG $^{1 *}$, MARIAM A.M. AL-BEITI ${ }^{1 *}$, JUNCHEN WANG $^{2}$, GUANGWEI WEI $^{3}$, \\ JUN LI ${ }^{1}$, SHANHUI LIANG ${ }^{1}$ and XIN LU ${ }^{1}$ \\ ${ }^{1}$ Department of Gynecology, Obstetrics and Gynecology Hospital, Fudan University, Shanghai 200011; \\ ${ }^{2}$ Department of Pathology, East Hospital of Tongji University, Shanghai 200120; ${ }^{3}$ Department of Anatomy, \\ Shandong University, Jinan, Shandong 250012, P.R. China
}

Received August 3, 2011; Accepted February 6, 2012

DOI: $10.3892 / \mathrm{mmr} .2012 .810$

\begin{abstract}
Homeodomain-interacting protein kinase 2 (HIPK2) is a serine/threonine nuclear kinase that is involved in apoptosis and cell growth, and is also thought to play a role in the process of tumorigenesis. The purpose of this study was to identify the role of HIPK 2 in cervical cancer. HIPK 2 expression was examined in normal and cervical cancer tissues at the mRNA and protein levels by quantitative real-time PCR and western blotting. To investigate the mechanism of action of HIPK2 in cervical cancer, RNA interference was used to analyze the effect of HIPK2 on apoptosis and cell growth in cervical cell lines. The results showed that HIPK 2 expression was significantly higher in the cervical cancer tissues compared to the normal cervical tissues, both at the mRNA and protein level. Moreover, inhibition of HIPK2 promoted cell growth and decreased the rate of cell apoptosis in cervical cell lines. Taken together, these results indicate that HIPK2 expression is higher in cervical cancer tissues and has a positive correlation with cervical cancer. HIPK2 may be important in the development of cervical cancer.
\end{abstract}

\section{Introduction}

Cervical cancer is the second most common type of female cancer worldwide (1). Human papillomavirus (HPV) infection is widely regarded as the most significant risk factor in the development of almost all cases of cervical cancer (2-4). However, certain other genetic and epigenetic factors may contribute to cervical carcinogenesis (5).

Correspondence to: Professor Xin Lu, Department of Gynecology, Obstetrics and Gynecology Hospital, Fudan University, Shanghai 200011, P.R. China

E-mail: xinlu98@yahoo.com

*Contributed equally

Key words: homeodomain-interacting protein kinase 2, cervical cancer, RNA interference, apoptosis
Homeodomain-interacting protein kinase 2 (HIPK2) is a serine/threonine nuclear kinase and is well conserved in various organisms (6). HIPK2 is localized mainly in the nuclei and has been mapped to chromosome 7q32-q34 $(7,8)$, which is known to be frequently rearranged in certain human neoplasms. HIPK2 regulates apoptosis, cell growth, proliferation and gene transcription. It was found to be expressed in human tissues, including blood marrow, lymph nodes, pancreas, spleen, thymus, heart, muscle and kidney. HIPK2 expression is decreased in the majority of human breast and thyroid carcinomas (7). Moreover, the loss of heterozygosity observed at chromosome 7q32-q33 in some neoplasms suggests that HIPK2 is a candidate tumor suppressor gene (7). However, overexpression of HIPK2 was found in colorectal cancer (9). These data demonstrated that HIPK2 may play different roles in various types of human cancer.

Previously, we documented overexpression of the HIPK2 protein in cervical cancer by immunohistochemistry (10). The expression was significantly associated with clinical stage. To investigate the role and biological function of HIPK2 in cervical cancer, RNA interference (RNAi) technology was used to silence HIPK2 expression in cervical cancer cell lines and determine the effect of HIPK2 on apoptosis and cell growth.

\section{Materials and methods}

Clinical samples and cell culture. Fresh surgical specimens were obtained from 20 cervical cancer patients admitted to the Obstetrics and Gynecology Hospital of Fudan University from January 2008 to January 2009 (Table I). None of the patients with cervical cancer had a previous history of chemotherapy and radiotherapy. Normal cervical tissues were also obtained from 10 patients with uterine myoma, which were used as controls. This study was approved by the ethics committee of the Obstetrics and Gynecology Hospital at Fudan University, China, and all patients provided written informed consent.

The human cervical cancer cell line, CaSki, was purchased from the American Type Culture Collection (ATCC, Manassas, VA, USA). Cell lines were maintained in RPMI-1640 (Gibco, Carlsbad, CA, USA), supplemented with $10 \%$ (v/v) fetal bovine serum (FBS; Gibco) and $100 \mathrm{U} / \mathrm{ml}$ antibiotics (Gibco) in a humidified incubator at $37^{\circ} \mathrm{C}$ with $5 \% \mathrm{CO}_{2}$. 
Reverse transcription PCR (RT-PCR) and quantitative real-time PCR analysis. Total RNA was extracted from the fresh tissue samples and the cervical cancer cell lines using TRIzol (Invitrogen, Carlsbad, CA, USA); RNA was reversetranscribed into cDNA using a reverse transcription kit (MBI Fermentas, Flamborough, Canada). The specificity of products of RT-PCR was identified by agarose gel electrophoresis.

SYBR-Green real-time PCR mix (Takara, Japan) was used to analyze the mRNA level, followed by quantification, normalization to $\beta$-actin and statistical analysis. The thermal profile was $95^{\circ} \mathrm{C}$ for $3 \mathrm{~min}$, followed by 40 cycles of $95^{\circ} \mathrm{C}$ for $15 \mathrm{sec}, 60^{\circ} \mathrm{C}$ for $15 \mathrm{sec}$ and $70^{\circ} \mathrm{C}$ for $30 \mathrm{sec}$. The expression level of mRNA was analyzed using the $2^{-\Delta \Delta C T}$ method. The real-time PCR primer set for HIPK2 was: Human HIPK2, forward: 5'-CTGTTTGTGTGGTGGTGTTG-3'; and reverse: 5'-GAGAGATTCGTTTGGAGATTGG-3'. In addition, a set of primers for $\beta$-actin, forward: 5'-GTGGACATCCGCAAA GAC-3', and reverse: 5'-AAAGGGTGTAACGCAACTA-3', was used to serve as an internal control for the amount of cDNA tested.

Western blot analysis. Whole protein was extracted from the fresh tissue samples as well as the cervical cancer cell lines, and equal amounts were separated on $8 \%$ SDS-PAGE gels and transferred onto polyvinylidene difluoride membranes. After blocking in $10 \%$ milk in PBS-T for $1 \mathrm{~h}$ at room temperature, membranes were incubated with HIPK2 antibody (Abcam Ltd., Cambridge, UK) overnight at $4^{\circ} \mathrm{C}$. The primary antibodies for HIPK2 and $\beta$-actin (Santa Cruz Biotechnology, Santa Cruz, CA, USA) were diluted according to the manufacturer's instructions. Following incubation with anti-mouse or anti-rabbit secondary antibody (Abcam Ltd.) for $1 \mathrm{~h}$ at room temperature, the results were detected using enhanced chemiluminescence (Pierce, Rockford, IL, USA) and exposure to X-ray film.

RNAi. The siRNA reagents used included siGENOME SMARTpool siRNA for human HIPK2 (catalog no. M-00326603-0005) and siGENOME RISC-Free Control siRNA (catalog no. D-001220-01-05) for a negative control (Thermo Fisher Scientific, MA, USA). Cervical cancer cell lines were cultured in antibiotic-free complete medium and transfected according to the manufacturer's instructions. Each experiment included the following samples: Untreated cells; negative control cells; and HIPK2 RNAi cells. Cells were incubated in $37^{\circ} \mathrm{C}$ with $5 \% \mathrm{CO}_{2}$ for $48 \mathrm{~h}$ for the mRNA and protein analysis to test the transfection efficiency. The trials were repeated at least 3 times independently.

Apoptosis analysis. Following transfection for $48 \mathrm{~h}$, cisplatinum (CaSki cells $2 \mu \mathrm{g} / \mathrm{ml}$ based on the IC50 dose) was added $24 \mathrm{~h}$ prior to harvesting to induce apoptosis. Cells were harvested using trypsin (EDTA-free), washed in cold PBS and collected by centrifugation ( $5 \mathrm{~min} ; 1,000 \mathrm{rpm}$ ). To detect the apoptotic cells, collected cells were resuspended in binding buffer, incubated with propidium iodide (PI) and Annexin-V. Following $15 \mathrm{~min}$ at room temperature in the dark, samples were measured by flow cytometry with FACSCalibur (Becton, Dickinson and Co., Franklin Lakes, NJ, USA) in triplicate. At least 10,000 cells were analyzed per sample.
Table I. Clinicopathological data.

\begin{tabular}{lcc}
\hline Patient characteristics & $\mathrm{n}$ & $\%$ \\
\hline Age (year) & & \\
Median & 45.65 & \\
Range & $(33-68)$ & \\
Stage & & 80 \\
I & 16 & 20 \\
II & 4 & \\
Histology & & 100 \\
Squamous & 20 & 0 \\
Adenocarcinoma & 0 & 20 \\
Grade & & 75 \\
1 & 4 & 5 \\
2 & 15 & \\
3 & 1 & \\
\hline
\end{tabular}

Cell growth analysis. Cell proliferation was evaluated using the methyl-thiazoldiphenyl tetrazolium (MTT) assay. Cells were cultured, trypsinized and seeded into 96-well culture plates (2,000 cells per well) 10 times. Cell growth was determined following transfection for $48 \mathrm{~h}$. In brief, $20 \mu \mathrm{l} \mathrm{MTT}(5 \mathrm{mg} / \mathrm{ml})$ was added to each well and cells were maintained at $37^{\circ} \mathrm{C}$ in a humidified incubator with $5 \% \mathrm{CO}_{2}$ for $4 \mathrm{~h}$. Cultured cells were lysed by $100 \mu \mathrm{l}$ DMSO to measure the absorbance at $570 \mathrm{~nm}$.

Statistical analysis. Statistical evaluations were performed using SPSS 16.0 (IBM, Chicago, IL, USA) for Windows. Experiment results were tested by the paired-samples t-test. $\mathrm{P}<0.05$ was considered to indicate a statistically significant difference and was presented as two-tailed.

\section{Results}

Expression of HIPK2 in cervical tissues. The clinical characteristics of the cervical cancer patients are shown in Table I. Expression of HIPK 2 mRNA was investigated by quantitative real-time PCR. We found that HIPK2 mRNA was significantly higher in cervical cancer than in normal cervical tissues $(\mathrm{P}<0.05)$ (Fig. 1A). HIPK2 protein was detected by western blot analysis and the results corresponded with those of HIPK2 mRNA expression (Fig. 1B).

Expression of HIPK2 suppressed by RNAi in cervical cancer cells in vitro. Following transfection for $48 \mathrm{~h}$, the efficiency of HIPK2 RNAi was verified to produce at least a $75 \%$ decrease by assessing the mRNA silencing of negative control RNAi. The expression of protein and mRNA of HIPK2 in cervical cell lines was tested by western blotting (Fig. 2A) and quantitative real-time PCR (Fig. 2B). Based on this, $50 \mathrm{pmol} / \mathrm{ml}$ siRNA was used in suppressing the expression of HIPK2 in cervical cancer lines.

Apoptosis analysis in cervical cancer cells with HIPK2 knockdown. Apoptotic cells (Annexin- $\mathrm{V}^{+} / \mathrm{PI}^{-}$) were detected by 
A

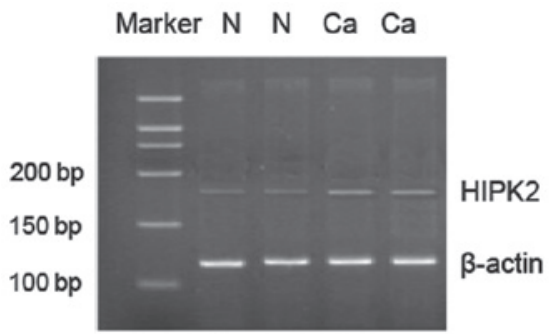

C

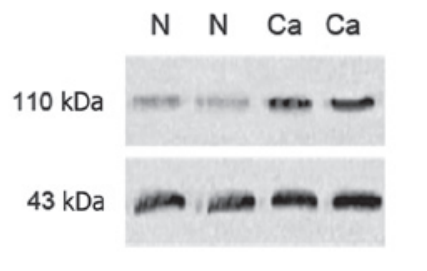

B

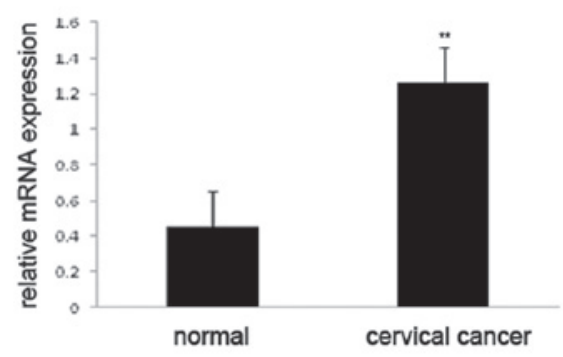

D

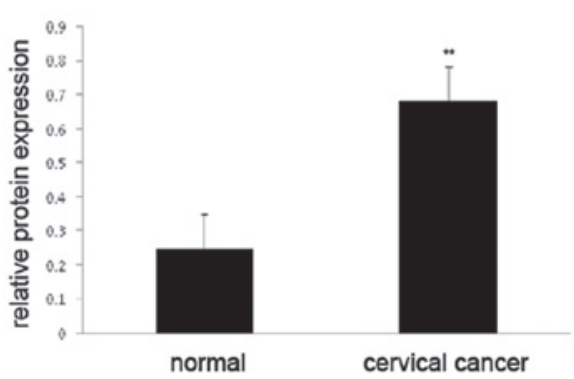

Figure 1. (A) Expression of HIPK2 in two normal (N) and two cervical cancer (Ca) tissues in agarose gel by RT-PCR. (B) Quantitative real-time PCR of HIPK2 mRNA expression. (C and D) Western blot analysis of HIPK2 protein expression, $\beta$-actin was used as a loading control. ${ }^{* *} \mathrm{P}<0.05$ versus normal cervical tissues. RT-PCR, reverse transcription PCR; HIPK2, homeodomain-interacting protein kinase 2.

A

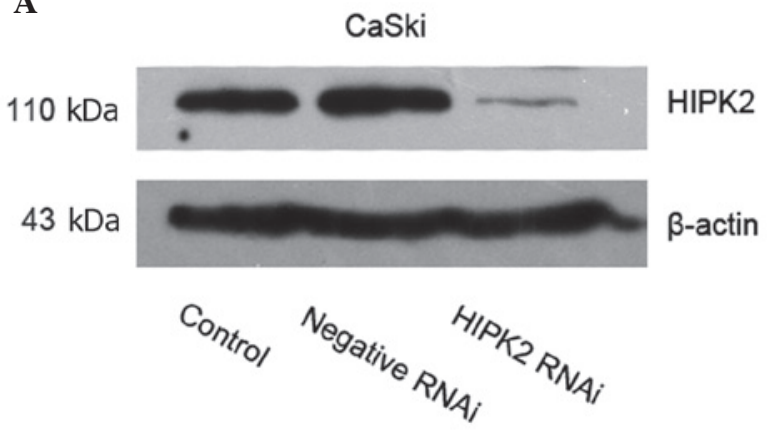

B

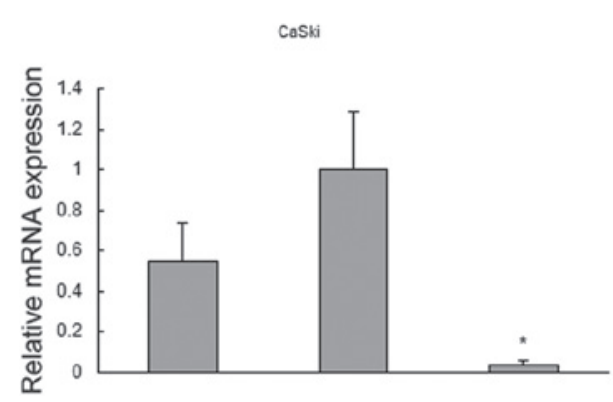

Figure 2. HIPK2 expression in CaSki pre- and post-RNAi. Cell lines were incubated with transfection reagent only (Control), transfected with a RISC-free control siRNA (Negative RNAi), or siRNA for HIPK2 (HIPK2 RNAi). (A) HIPK2 protein expression was tested by western blot analysis; $\beta$-actin was used as a loading control. (B) HIPK2 mRNA expression was analyzed by quantitative real-time PCR, and at least a 75\% decrease in mRNA level in the HIPK2 RNAi group versus the negative control RNAi group was observed. ${ }^{*} \mathrm{P}<0.01$ versus the negative control. RNAi, RNA interference; HIPK2, homeodomain-interacting protein kinase 2.

flow cytometry following Annexin-V/PI staining. Our results showed that the apoptosis rate of cells transfected with siRNA for human HIPK2 was higher than that of the negative control or untreated cells in CaSki cell lines (Fig. 3).
Cell growth analysis in cervical cancer cells with HIPK2 silencing. An MTT assay was used to evaluate cell growth. Cells transfected with siRNA for HIPK2 showed a significant decrease in cell growth compared with negative controls and controls in CaSki cells (Fig. 4).

\section{Discussion}

HIPK 2 protein is a member of a recently identified family of nuclear protein kinases that interact with homeodomain transcription factors and that are well conserved in various organisms $(6,8)$. HIPK2 expression in humans is lower, with a relatively strong expression restricted to heart, muscle and kidney. HIPK2 is known to be reduced in the majority of human breast and thyroid carcinomas (7). However, the overexpression of HIPK2 was found in colorectal cancer patients with familial adenomatous polyposis (9).

In a previous study, we documented the increased expression of HIPK 2 protein in cervical cancer, which was detected by immunohistochemistry (10). This expression was significantly associated with advanced clinical stages. In the present study, we have found that the expression levels of HIPK 2 mRNA and protein were significantly higher in cervical cancer than those in normal cervical tissues. These findings indicate that HIPK2 is important in cervical carcinogenesis.

HIPK2 is an essential factor that regulates cell growth and apoptosis in normal tissues and certain neoplasms. Apoptosis is a distinct mode of cell death that is responsible for deletion of cells in normal tissues. It also occurs spontaneously in malignant tumors, often markedly retarding their growth (11). HIPK 2 may be regulated by the p53 tumor suppressor gene (12). Several studies have shown that HIPK2 kinase function enhances the expression of wild-type p53 (WTp53) target genes, resulting in growth arrest and the enhancement of apoptosis (13-17). In the present study, in order to elucidate the 
A

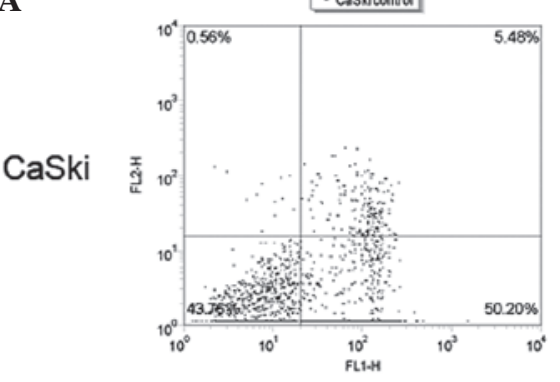

Control

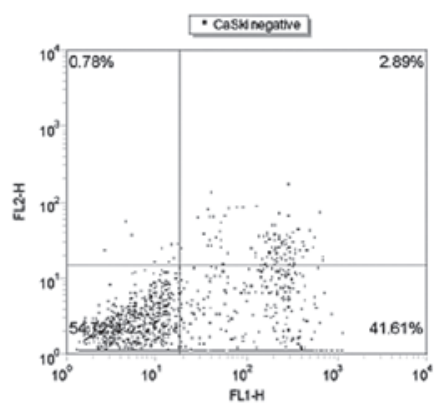

Negative RNAi



HIPK2 RNAi

B

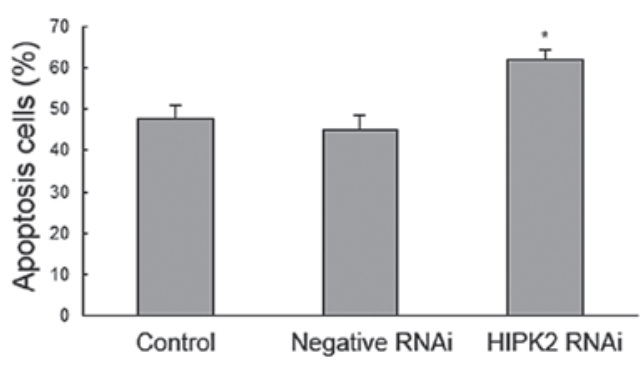

Figure 3. (A) Apoptosis and death levels of CaSki cells $48 \mathrm{~h}$ following treatment as detected by FACS after Annexin-V/PI staining is shown. Apoptotic (Annexin- $\mathrm{V}^{+} / \mathrm{PI}{ }^{-}$) populations were readily identified by FL1-H and $\mathrm{FL} 2-\mathrm{H}$. (B) Statistical evaluations of FACS show the increased rate of apoptosis in HIPK2 RNAi cells. ${ }^{*} \mathrm{P}<0.01$ versus negative control. FACS, fluorescence-activated cell sorting; PI, propidium iodide; HIPK2, homeodomain-interacting protein kinase 2 .

role of HIPK2 in cervical cancer, RNAi was used to silence the HIPK2 expression. In normal circumstances, we found that the cervical cancer cell line has a low rate of apoptosis $(\leq 6 \%)$. To avoid measuring error and to enlarge the rate of apoptosis, cisplatinum was employed at a dose of IC50 in untreated cells,

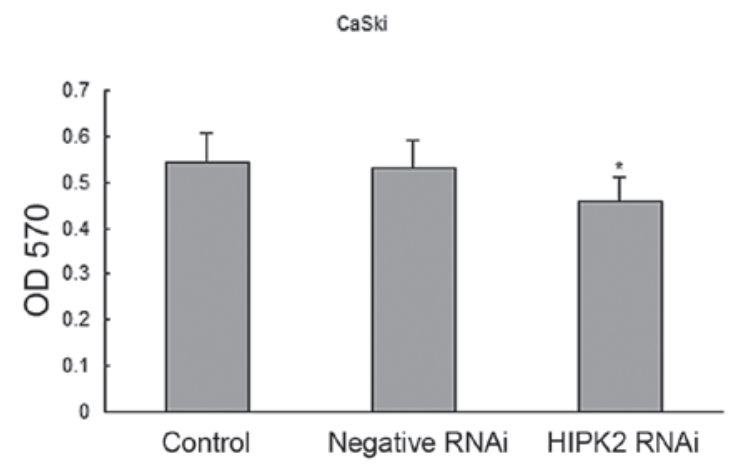

Figure 4. The effect of HIPK2 RNAi on the cell growth of CaSki cells was analyzed by MTT assay. Cells transfected with siRNA for HIPK2 show an increase rate of cell growth compared with the negative control and the cervical cell line controls. ${ }^{*} \mathrm{P}<0.01$ versus negative control. HIPK2, homeodomain-interacting protein kinase 2.

and the results of flow cytometry showed that the silencing of HIPK2 increases apoptosis in cervical cancer cells. Results of the MTT assay showed that more cells are present in wells following HIPK2 interference, indicating that a low HIPK2 expression is correlated with a low cell growth rate. Besides, a significant toxic effect was not observed in tests using the transfection reagent. In our previous study, no association between HIPK2 expression and the histological type (10) was found; thus, we only selected 20 cases of squamous cervical cancer for the expression study. In this study, we also found a correlation between high-risk HPV and HIPK2 in cervical cancer (data not shown). However, the specific role of HIPK2 with HPV has not been identified, and further studies of HPV E6/E7 protein and HIPK2 are required.

In conclusion, we have demonstrated that HIPK2 expression was significantly higher in cervical cancer than in normal cervical tissues. Additionally, HIPK2 interference demonstrated that HIPK2 was involved in the regulation of cell apoptosis and growth in cervical cancer cells. There is a positive correlation between the expression of HIPK2 and cervical cancer. HIPK2 may therefore be involved in the development of cervical cancer, and may be a new tumor marker in cervical cancer.

\section{Acknowledgements}

This study was supported by grants from the Natural Science Foundation of China (No. 30772311).

\section{References}

1. Schiller JT and Lowy DR: Prospects for cervical cancer prevention by human papillomavirus vaccination. Cancer Res 66: 10229-10232, 2006.

2. Muñoz N, Bosch FX, de Sanjosé S, et al: Epidemiologic classification of human papillomavirus types associated with cervical cancer. N Engl J Med 348: 518-527, 2003.

3. Parkin DM and Bray F. Chapter 2: the burden of HPV-related cancers. Vaccine 24: S11-S25, 2006.

4. Vinokurova S, Wentzensen N, Kraus I, et al: Type-dependent integration frequency of human papillomavirus genomes in cervical lesions. Cancer Res 68: 307-313, 2008.

5. Lazo PA: The molecular genetics of cervical carcinoma. Br J Cancer 80: 2008-2018, 1999. 
6. Kim YH, Choi CY, Lee SJ, Conti MA and Kim Y: Homeodomaininteracting protein kinase, a novel family of corepressors for homeodomain transcription factors. J Biol Chem 273: 875-879, 1998.

7. Pierantoni GM, Bulfone A, Pentimalli F, et al: The homeodomain-interacting protein kinase 2 gene is expressed late in embryogenesis and preferentially in retina, muscle, and neura tissues. Biochem Biophys Res Commun 290: 942-947, 2002.

8. Wang Y, Hofmann TG, Runkel L, et al: Isolation and characterization of cDNAs for the protein kinase HIPK2. Biochim Biophys Acta 1518: 168-172, 2001.

9. D'Orazi G, Sciulli MG, Di Stefano V, et al: Homeodomaininteracting protein kinase-2 restrains cytosolic phospholipase A2-dependent prostaglandin E2 generation in human colorectal cancer cells. Clin Cancer Res 12: 735-741, 2006.

10. Al-Beiti M and Lu X: Expression of HIPK2 in cervical cancer: correlation with clinicopathology and prognosis. Aust $\mathrm{N} \mathrm{Z} \mathrm{J}$ Obstet Gynaecol 48: 329-336, 2008.

11. Pierantoni GM, Fedele M, Pentinalli F, et al: High mobility group I (Y) proteins bind HIPK2, a serine-threonine kinase protein which inhibits cell growth. Oncogene 20: 6132-6141, 2001.
12. Dauth I, Krüger J and Hofmann TG: Homeodomain-interacting protein kinase 2 is the ionizing radiation-activated p53 serine 46 kinase and is regulated by ATM. Cancer Res 67: 2274-2279, 2007.

13. Levine AJ: p53, the cellular gatekeeper for growth and division. Cell 88: 323-331, 1997.

14. Hofmann TG, Moller A, Sirma H, et al: Regulation of p53 activity by its interaction with homeodomain-interacting protein kinase 2. Nat Cell Biol 4: 1-10, 2002.

15. D'Orazi G, Cecchinelli B, Bruno T, et al: Homeodomaininteracting protein kinase 2 phosphorylates p53 at Ser 46 and mediates apoptosis. Nat Cell Biol 4: 11-19, 2002.

16. Wang Y, Debatin KM and Hug H: HIPK2 overexpression leads to stabilization of p53 transcriptional activity by decreasing Mdm2 protein levels. BMC Mol Biol 2: 8, 2001.

17. Gresko E, Roscic A, Ritterhoff S, Vichalkovski A, Sal G and Schmitz ML: Autoregulatory control of the p53 response by caspase-mediated processing of HIPK2. EMBO J 25: 1883-1894, 2006. 